\title{
Perhalophenyltetrahydrothiophenegold(I) as
}

\section{Lewis Base in Acid-Base reactions with Silver}

\section{trifluoroacetate.}

Eduardo J. Fernández ${ }^{\S}$, Peter G. Jones, ${ }^{\#}$ Antonio Laguna**, José M. López-deLuzuriaga $^{\S}$, Miguel Monge ${ }^{\S}$, M. Elena Olmos ${ }^{\S}$ and Raquel C. Puelles ${ }^{\S}$

Departamento de Química. Universidad de la Rioja. Grupo de Síntesis Química de La Rioja, UA-CSIC. Complejo Científico Tecnológico. 26006 Logroño (Spain).

Departamento de Química Inorgánica. Instituto de Ciencia de Materiales de Aragón, Universidad de Zaragoza-CSIC. 50009 Zaragoza (Spain).

Institüt für Anorganische und Analytische Chemie der Technischen Universität, Postfach 3329, D-38023 Braunschweig (Germany). 


\section{TD-DFT RESULTS}

MODEL 1A [Au (C6F5) (tht) ] $2 \cdots[A g 2(\mathrm{CF} 3 \mathrm{CO} 2) 2]$

FIRST AND SECOND TRIPLET EXCITATIONS. HOMO is 168a orbital; LUMO is 169 a orbital

Excitation energies and oscillator strengths:

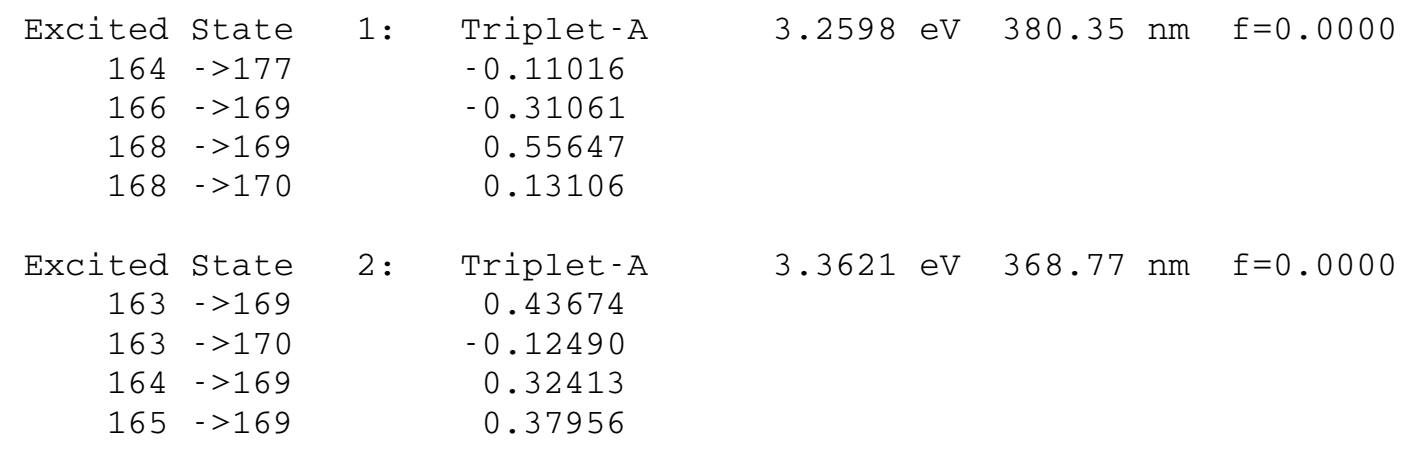

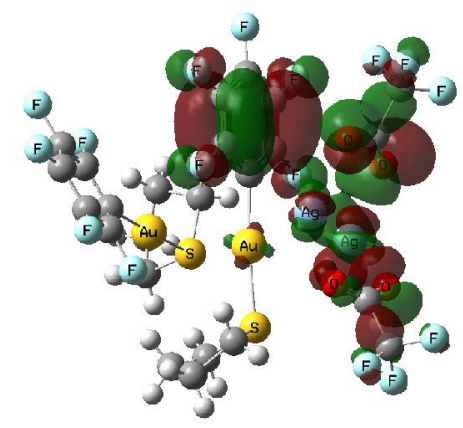

HOMO - 5

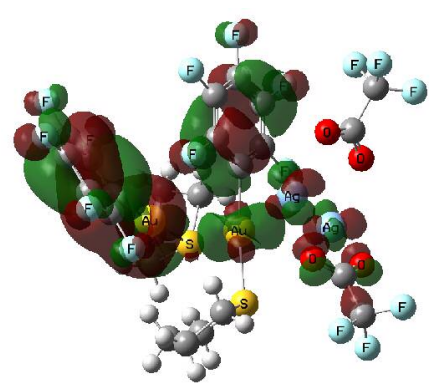

HOMO - 2

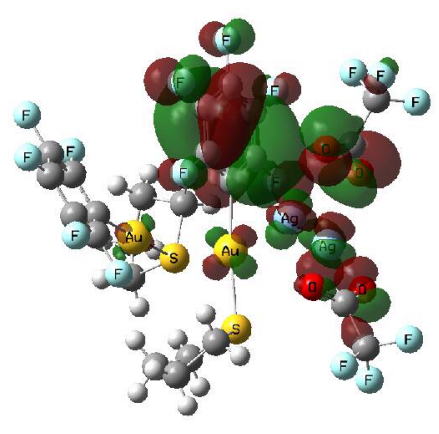

HOMO - 4

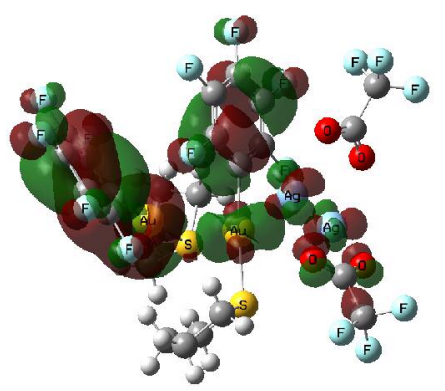

HOMO

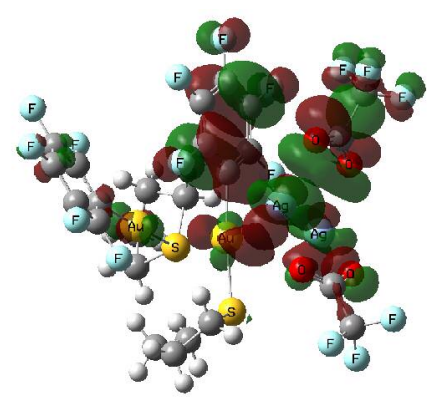

HOMO - 5

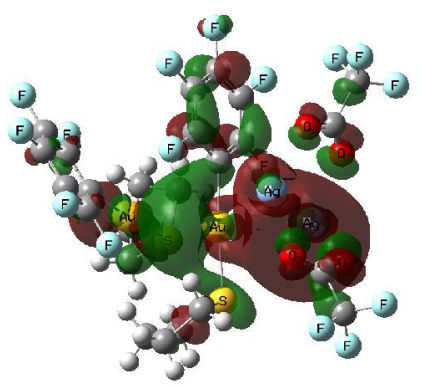

LUMO 
FIRST TRIPLET EXCITATION. HOMO is 114a orbital; LUMO is 115a orbital

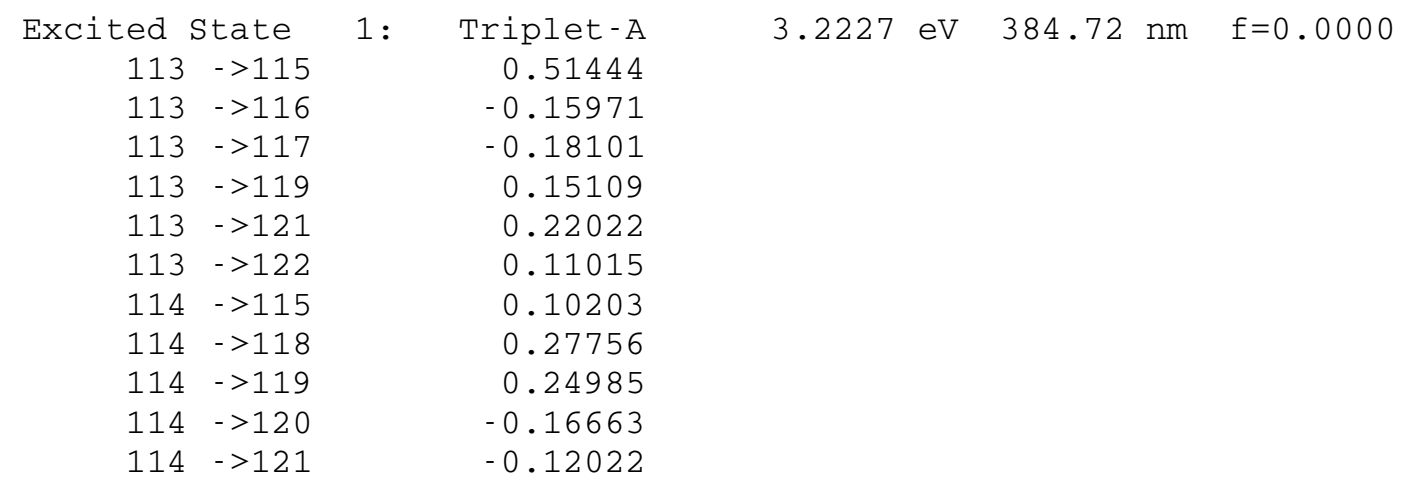
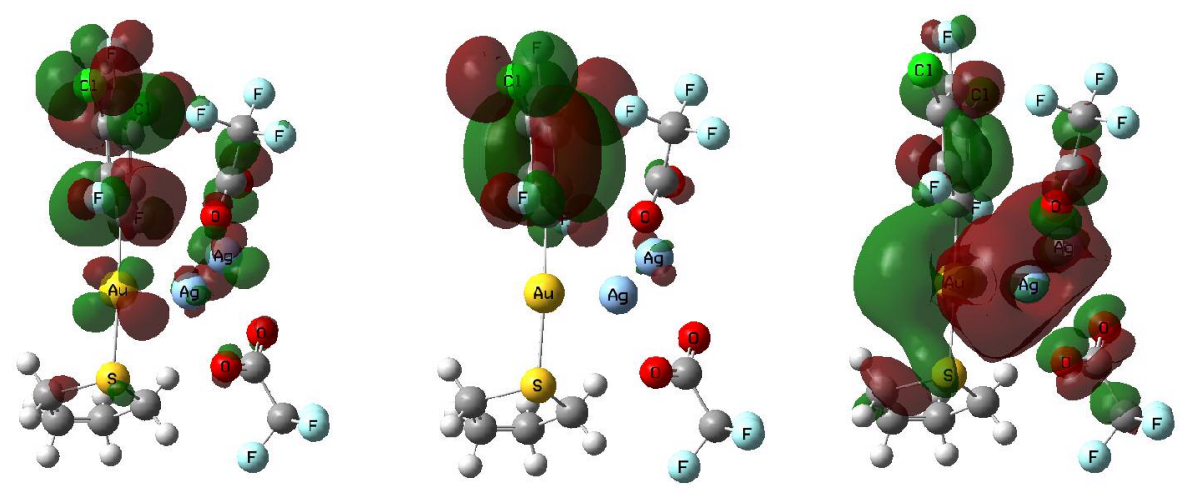

HOMO - 1

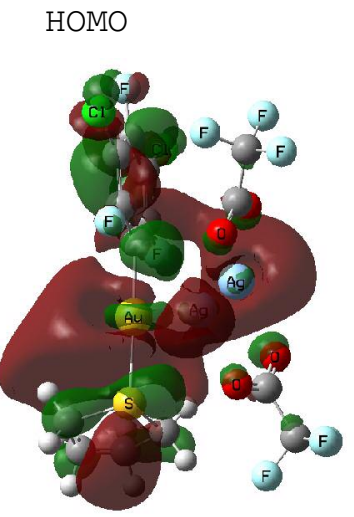

LUMO

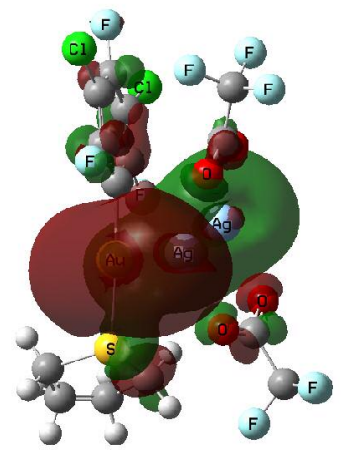

$\mathrm{LUMO}+2$

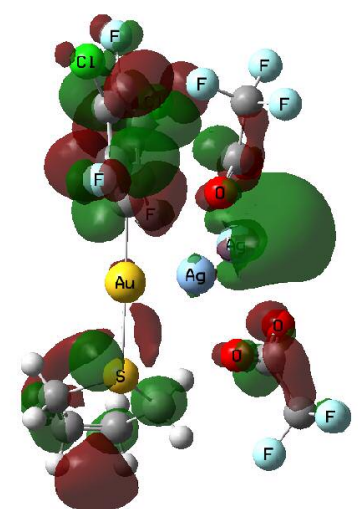

LUMO+4

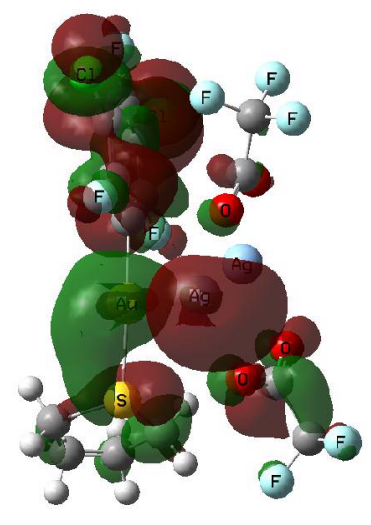

LUMO+5
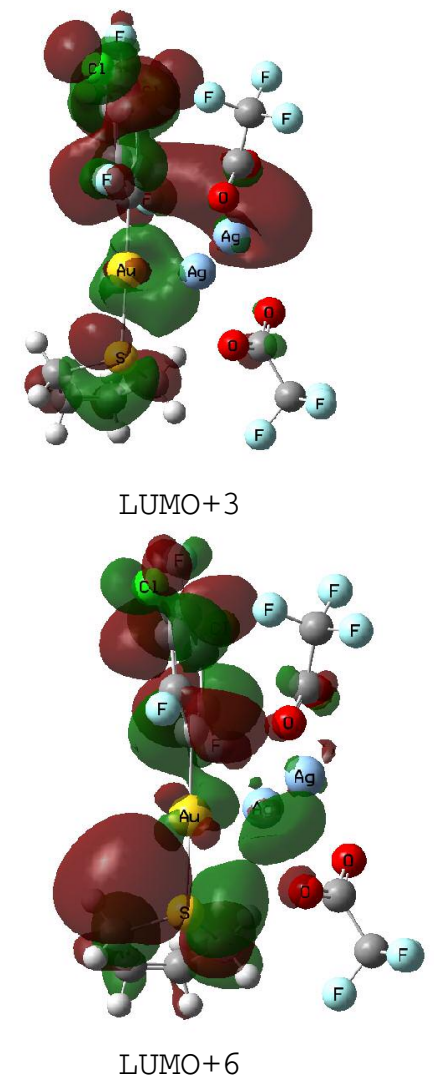


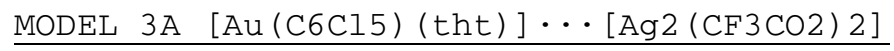

FIRST TRIPLET EXCITATION. HOMO is 114a orbital; LUMO is 115a orbital Excitation energies and oscillator strengths:

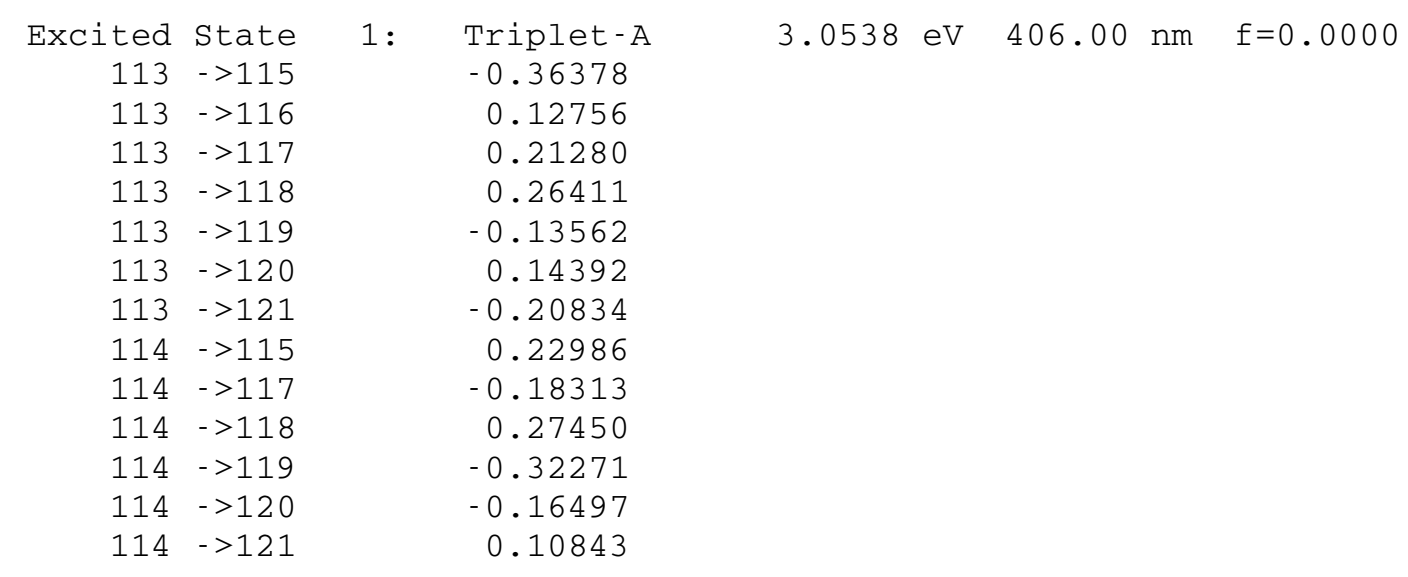

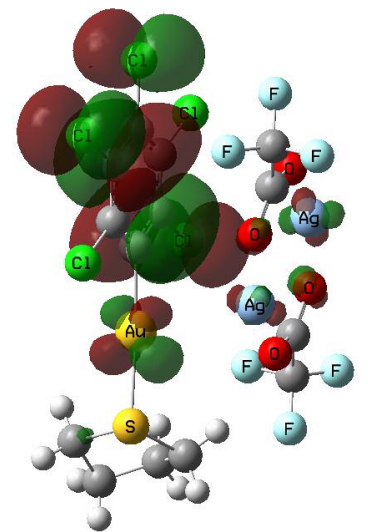

HOMO - 1

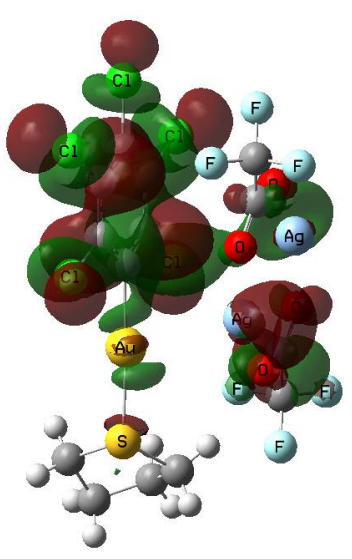

LUMO+3

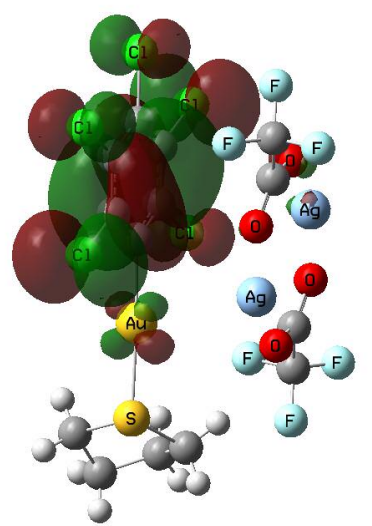

HOMO

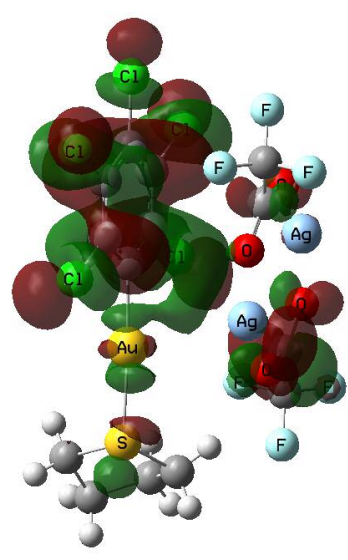

LUMO +4

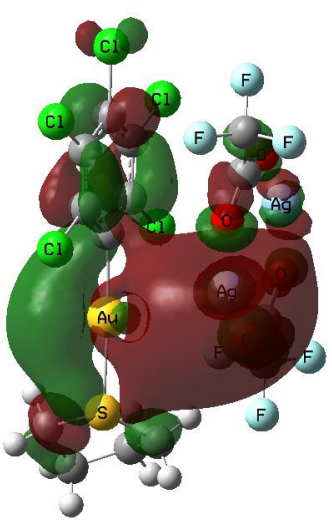

LUMO 